Provided for non-commercial research and education use. Not for reproduction, distribution or commercial use.

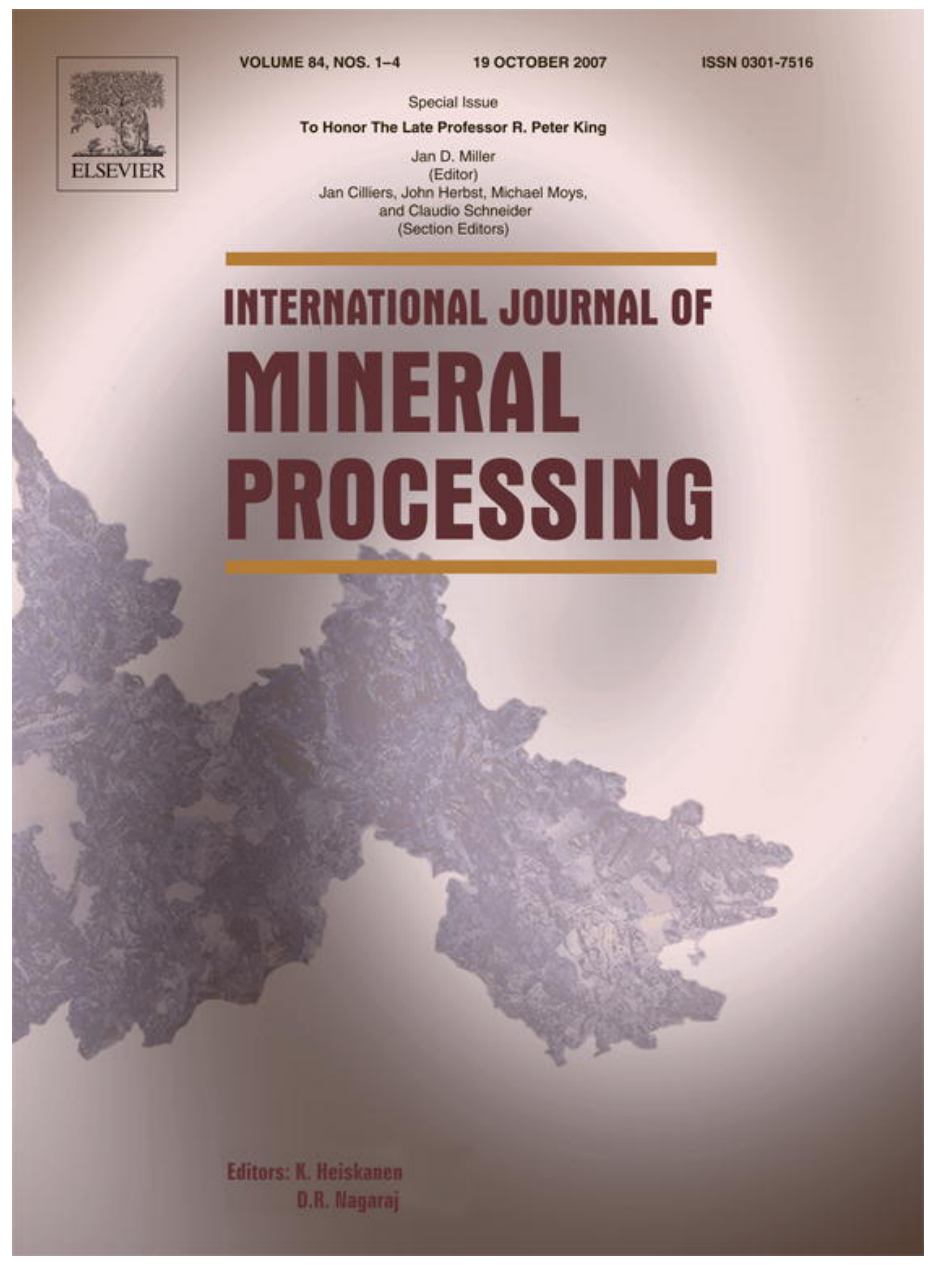

This article was published in an Elsevier journal. The attached copy

is furnished to the author for non-commercial research and education use, including for instruction at the author's institution, sharing with colleagues and providing to institution administration.

Other uses, including reproduction and distribution, or selling or licensing copies, or posting to personal, institutional or third party websites are prohibited.

In most cases authors are permitted to post their version of the article (e.g. in Word or Tex form) to their personal website or institutional repository. Authors requiring further information regarding Elsevier's archiving and manuscript policies are encouraged to visit: 


\title{
Particle texture analysis using polarized light imaging and grey level intercepts
}

\author{
Eric Pirard *, Sophie Lebichot, William Krier \\ Université de Liège, GeMMe-MICA: Georesources and Geo-Imaging Group, Sart Tilman B52, 4000 LIEGE, Belgium
}

Received 31 March 2006; received in revised form 21 February 2007; accepted 12 March 2007

Available online 24 March 2007

\begin{abstract}
The usual characterization of particulate ore material with image analysis includes modal analysis and liberation analysis. Both methods include stereological corrections based on intercept length distributions within each phase of interest. The first principle of stereology relies on a simple assumption of uniform random sectioning, whereas the liberation models require all particles to have similar textural properties. However, for the sake of adequate prediction of ore behavior in industrial processes it is becoming more and more important to be able to describe particle populations in terms of texture classes.

Among the texture analysis techniques, this paper focuses on mean intercept length (MIL). It brings two important improvements: the first one is to improve grain boundary imaging by using images of bireflectant minerals under plane polarized illumination, the second one is to consider intercept analysis on grey level images instead of binary ones. The method is illustrated on a selection of critical hematite textures found within banded iron formations.
\end{abstract}

(C) 2007 Elsevier B.V. All rights reserved.

Keywords: Microscopy; Intercepts; Stereology; Texture; Bireflectance; Iron ore

\section{Introduction}

\subsection{Image analysis of particulate ore material}

Since the very first attempts by Delesse, the development of analytical mineralogy of rocks and ores has been given considerable attention. Despite the availability of sophisticated photon, electron or X-ray microscopes and the continuous development of new sensors, there still appears to be a long way to go from a hand

\footnotetext{
* Corresponding author. Tel.: +32 436637 99; fax: +32 43669520 .

E-mail addresses: eric.pirard@ulg.ac.be (E. Pirard), sophie.lebichot@ulg.ac.be (S. Lebichot),w.krier@ulg.ac.be (W. Krier).
}

specimen to its full description in terms of contained mineral phases and textures.

The first challenge is the automated identification of mineral species on a pixel by pixel basis. This still faces major limitations but can be addressed using, or combining, energy dispersive X-ray spectra (Gottlieb et al., 2000), backscattered electron intensities (Gu, 2003) or specular reflectance spectra (Pirard, 2004). The resulting mineral mappings typically serve as an input to the first principle of stereology to straightforwardly derive the volumetric ratios of minerals from planar ratios. The accuracy of volumic ratio estimations is dependent upon the number, but also critically upon the randomness of cross-sections through the specimen (Isotropic Uniform Random (IUR) sections - Russ and Dehoff, 2001), a topic which is rarely discussed and validated in the 
geological/mineralogical image analysis literature. When dealing with particulate ores, the randomness prerequisite appears easier to satisfy because of the large number of particles involved. But, in many cases, severe segregation appears during powder handling and preparation of polished sections. Recent results obtained from reconstituted flotation residues with known mineral compositions have shown significant discrepancies (Mermillod-Blondin, 2005 ) that must be addressed by innovation in the sample preparation procedure in order to cope with preferential settling of heavy minerals in the epoxy resin.

Image analysis routines have brought a step forward in the global characterization of ores, but it is essentially the development of local analysis capabilities that will contribute to an unbeatable breakthrough in characterization technology. This is why a huge literature focuses on the characterization of individual particle properties and their distributions in terms of size, shape and liberation.

In this paper, special attention will be given to the estimation of textural properties. Texture is understood here in a broad sense: it relates both to the spatial distribution of different minerals and to the spatial distribution of different grains or crystals. In petrography and material sciences, many authors would privilege the word fabric analysis for the first kind of characterization and explicitly refer to texture analysis when it comes about quantifying preferred orientation in crystalline assemblages (Van der Pluijm and Marshak, 1997). An alternative term often used for texture in a general sense is microstructure, thereby clearly stating that the non-ending debate about what is texture and what is structure is useless as it very often depends on the scale of scrutiny that is being considered.

King and Schneider (1998) have compiled a series of data from the literature relating apparent linear liberation of both synthetic and natural particles with their true volumic grade. These data clearly demonstrate that there is no unique stereological transfer function and that the ore texture has a decisive influence on the liberation distribution. Fig. 1 displays a series of synthetic particles with identical apparent liberation ratios in 2D but different textures. The textural properties are either expressed by the spatial distribution of the mineral phases (particle a vs. particle b) or by the spatial arrangement of individual grains within each phase (particle b vs. particle c vs. particle d).

In practice, in order to develop quantitative textural analysis tools, it is essential to use or combine imaging techniques that provide sufficient contrast between individual mineral phases (mineralogical imaging) as well as between different grains or crystals within a single phase (crystallographic imaging). Because texture measurements are non-stereological (i.e. do not allow for the unbiased estimation of a property in $R^{n}$ from measurements performed in $R^{n-1}$ ) one could argue that imaging from sections has only limited interest and will be superseded by 3D imaging methods in the future (Lin and Miller, 1996). But, in the meanwhile, it is believed that the amount of information captured from 2D images is significant for industrial processes such as sintering, flotation, leaching, etc. wherein textural information is becoming more and more important to support conclusions and interpretations in process mineralogy.

\subsection{Imaging of mineral textures}

\subsubsection{Mineralogical imaging}

Despite a wide variety of imaging principles, it is obvious that no single system today is capable of capturing the extensive textural information. This would require a system able to locate any pixel on a high resolution 3D grid and give it both a chemical and a crystallographical attribute. Most research teams involved in modal analysis or liberation analysis of ores have adopted the Scanning Electron Microscope as their privileged imaging device (Gottlieb et al., 2000; Petruk, 1998). This allows for high resolution images to be collected either as single-valued back-scattered electron (BSE) images or as a multiple set of elementary mappings derived from the energy dispersive X-Ray spectrum of each pixel (EDX). The most
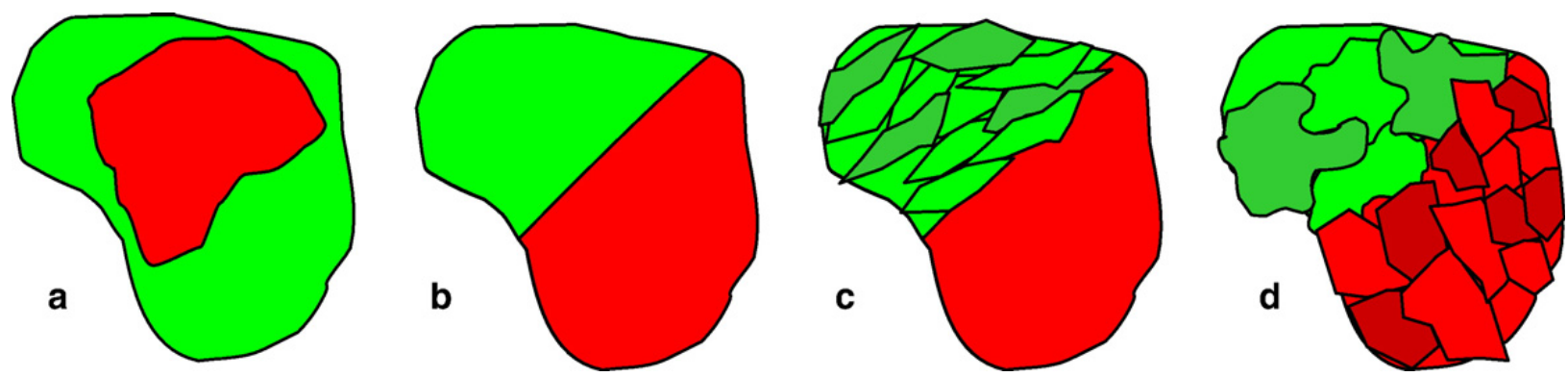

Fig. 1. Schematic representation of particles with identical apparent liberation ratios but different textural properties. Particles a and b differ in terms of spatial distribution of phases, whereas particles b, $\mathrm{c}$ and d differ in terms of spatial arrangement of grains. 
efficient and productive way being probably to couple both imaging systems: BSE helps to identify domains with different average atomic numbers, whereas punctual EDX spectra help to attribute compositional data to BSE pixels (Gu, 2003). Optical microscopy has often been disregarded because of its sensitivity to specimen preparation and its poor discriminative power (Petruk, 1988). This is particularly true with poor CCD sensors but can be significantly improved when using calibration and proper color imaging principles (Berrezueta, 2004).

The rapid development of desktop microfocus X-Ray computer tomography $(\mu \mathrm{CT})$ has popularized another imaging mode particularly suited for the quantitative analysis of porous materials in 3D (Cnudde et al., 2006; Ketcham and Iturrino, 2005). Up to now, phase discrimination based on desktop X-Ray absorption is still very poor. It is expected that future hardware developments might bring in mineral mapping capabilities and open the way to real phase boundary imaging in 3D.

\subsubsection{Crystal orientation imaging}

Since Adams et al. (1993) the semi-automatic analysis of diffraction patterns generated by back-scattered electrons is performed on a pixel by pixel basis to produce maps of crystallographic orientation. The technique is often designated under the term Orientation Imaging (OIM). This technique is based on band recognition and indexing that is only possible for selected mineral phases, previously identified by the operator and not suffering mis-indexing (Prior et al., 1999). Recent work published by Dingley (2004) illustrates how the phase identification retrieved from EDX spectra can be used as an input for OIM imaging of the same scene. The final result is a map wherein both phase boundaries and grain boundaries are visible. Despite its huge potential, such an imaging mode still requires tremendous computational efforts and has severe limitations. It is expected to become standard only by the end of the next decade.

\section{Texture imaging and analysis methods}

\subsection{Imaging of phase and grain boundaries in optical microscopy}

Reflected light microscopes use co-axial illumination to contrast individual minerals. Under such imaging conditions, every pixel of a digital image is characterized by a reflectance that is a function of the local surface properties and the intrinsic optical properties of the underlying mineral (absorption and refraction indices). Based on this, ore microscopists easily discriminate between most gangue minerals with very low reflectances $(<10 \%)$, oxide minerals with intermediate reflectances $(10 \%-30 \%)$ and sulfides which are typically higher $(15 \%-60 \%)$. The theory behind optical microscopy has been recently reviewed by Criddle (1998) and a quantitative database of reflectance spectra (QDF) for most opaque minerals has been compiled by Criddle and Stanley (1993). It is essential to remind that reflectance values are both a function of the wavelength and the crystal lattice orientation. Basically, isotropic minerals crystallizing in the cubic system are characterized by a unique reflectance spectrum (Fig. 2),

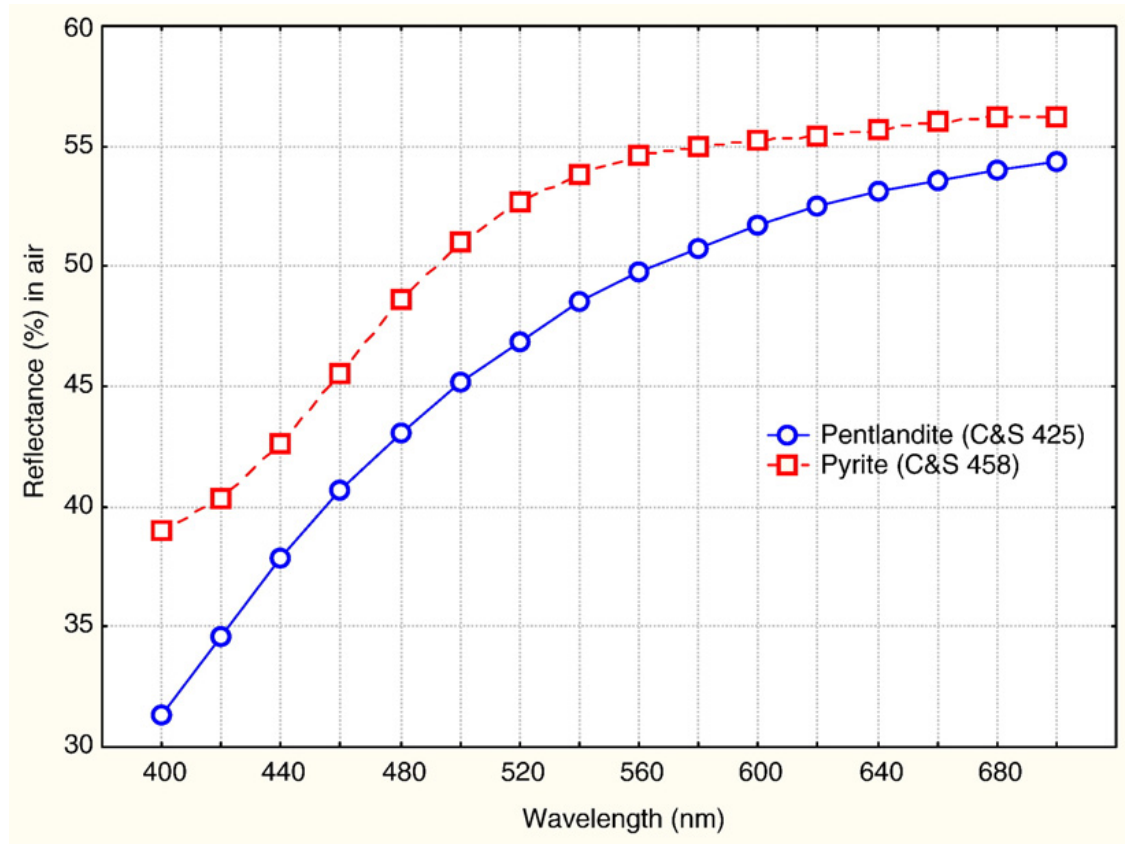

Fig. 2. Reflectance spectra plotted from the QDF database for Pentlandite (plain line) and Pyrite (dotted line). 
whereas uniaxial or biaxial crystals display a reflectance spectrum somewhere between a minimum $\left(R_{1}\right)$ and a maximum $\left(R_{2}\right)$ reflectance spectrum depending on crystal orientation (Fig. 3). Fig. 2 reveals why two cubic minerals such as pentlandite and pyrite are hard to identify for an untrained human eye. They have a very similar creamish yellow color and only differ in reflectance by a value of about $5 \%$. Fig. 3 shows why marcasite typically appears more whitish and less creamy than pyrite, but also evidences that stage rotation is often required to confirm marcasite by checking reflectance changes with crystal orientation (bireflectance property).

In order to grab the whole spectral information a correct imaging setup will take pictures of the mineral surface at different wavelengths and under different orientations of the specimen. Fig. 4 schematically represents such a system wherein a rotating filter wheel is located on the reflected light beam and allows the user to picture images at a selected range of wavelength using a Peltier cooled scientific grade CCD camera. The development of a multispectral imaging protocol taking calibrated reflectance measurements through interference filters with $10 \mathrm{~nm}$ bandwidth has been detailed in Pirard (2004). In that context, the analyzer had been removed and incident light was plane polarized parallel to the beamsplitter plate.

The complementary technique of multiradial imaging by making use of the polarized light has been used in this study and will be presented hereafter.

The combined use of a polarizer and an analyzer in perpendicular positions (crossed Nichols) is a classical means of determining the optical anisotropy of a mineral.
This principle has been exploited in digital imaging by Fueten (1997) who developed a specific rotating polarizer stage for the transmitted light microscope. The definite advantage of this technique is to avoid specimen rotation and subsequent warping operations to co-register images taken under different orientations. However, this elegant principle cannot be adopted sensu stricto in reflected light microscopy because the classical architecture relies on a beamsplitter plate that induces additional polarization. Hence, a rigorous imaging technique that would aim quantification of optical anisotropy should rely on specimen rotation, but this is rather cumbersome to combine with the use of a motorized stage aiming at efficient image analysis of a large number of particles.

When aiming at contrasting grains with different crystal lattice orientations and not at measuring optical anisotropy a simplified imaging mode can be adopted by rotating the sole incident beam polarizer. Under such a mode, despite the fact that the beamsplitter plate alters polarization, a significant amount of the bireflectance of a mineral surface can still be captured.

In practice, images are taken at a given wavelength when rotating the incident beam polarizer by steps of $15^{\circ}$ or less. All images are stacked together in such a way that a set of intensities is produced for every pixel of coordinates $(x, y)$ for each orientation $(\theta)$ of the polar:

$P_{(x, y)}=\left\{I_{(x, y)}^{\theta}\right\}$ for $\theta=0^{\circ} \ldots .180^{\circ}$

Bireflectance cannot be estimated from these values unless the intensities have been leveled in order to

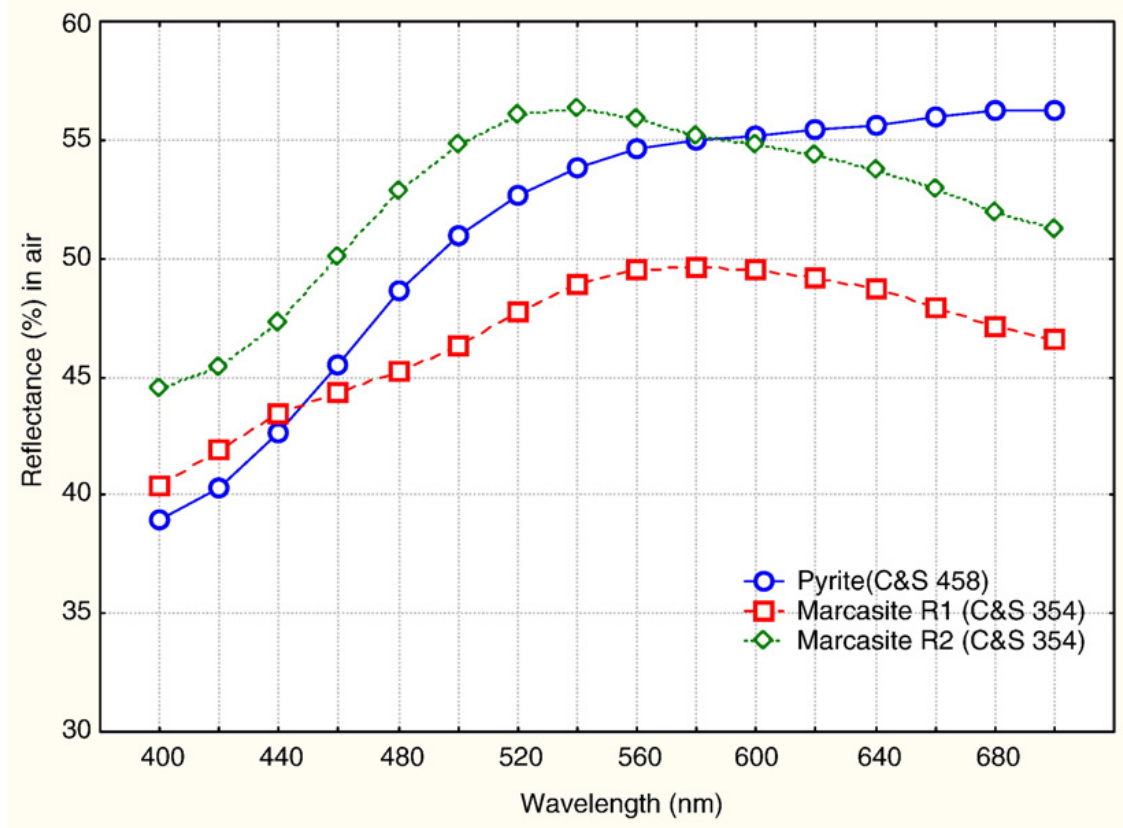

Fig. 3. Reflectance spectra plotted from the QDF database for Pyrite (plain line) and Marcasite ( two dotted lines indicating the bireflectance region). 


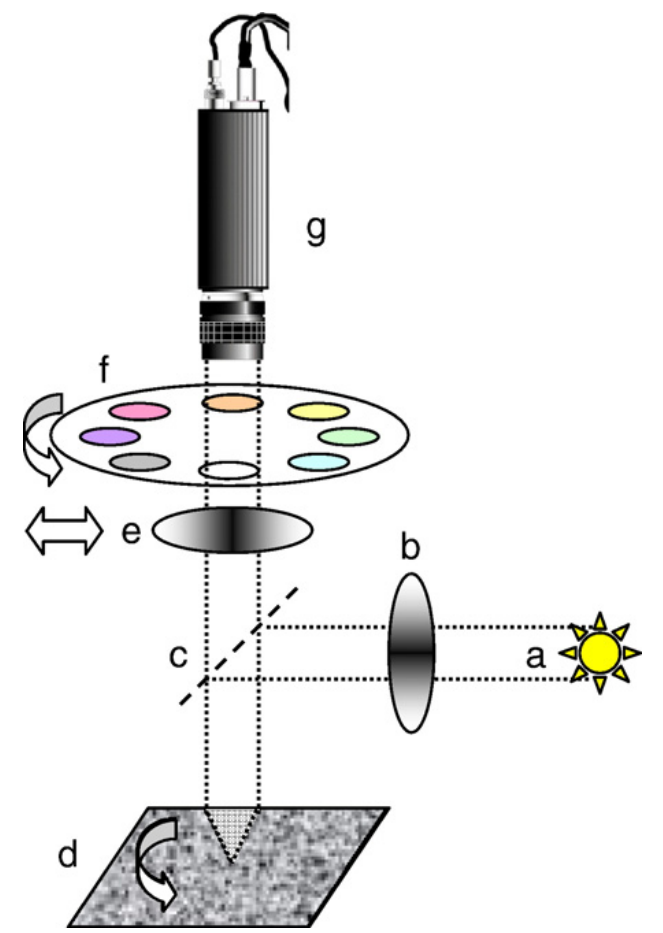

Fig. 4. Schematic representation of the optical path in a reflected light microscope fitted for crossed polar and multispectral imaging (a: halogen lamp; b: polarizer; c: beamsplitter plate; d: rotating sample stage; e: removable analyzer; f: rotating filter wheel; g: cooled 16 bit CCD camera).

compensate for the non constant intensity of the incident beam. This is due to the variable angle between the rotating incident beam and the fixed beamsplitter plate which cannot be avoided in a classical system. A simple way of coping with this is to equalize the average grey level intensity in each image. However, the strong hypothesis behind such leveling, which is not always satisfied, is that a sufficient number of crystals are present in the image and randomly oriented. An alternative and exact leveling method is to first identify an isotropic phase in the scene and use this as a grey level calibration feature from one image to the other. The resulting pixel values are now reflectances:

$P_{(x, y)}=\left\{R_{(x, y)}^{\theta}\right\}$ for $\theta=0^{\circ} \ldots . .180^{\circ}$

Fig. 5 illustrates a series of reflectance images at different polar orientations for three adjoining grains. Two of them are noticeably bireflectant whereas the third one appears isotropic (either a cubic crystal or a basal section of a uniaxial crystal).

Most minerals are bireflectant and not pleochroic, meaning thereby that the offset between the brightest reflectance $\left(R_{2}\right)$ and the lowest reflectance $\left(R_{1}\right)$ is almost constant throughout the spectrum. In case of pleochroism the multiradial imaging procedure should be repeated at several wavelengths and the variation in bireflectance from one wavelength to the other could be used as an additional identification criterion.

Posterior processing of the raw stack of oriented images consists in computing a set of three images that will support further identification of the individual mineral species and their grain boundaries.

A minimum reflectance image is obtained by computing the minimum reflectance on a pixel per pixel basis at each wavelength $(\lambda)$ over all orientations $(\theta)$ :

$R_{(x, y)}^{\lambda}=\min _{\theta}\left\{R_{(x, y)}^{\lambda, \theta}\right\}$

This image (Fig. 6a) is the ordinary reflectance spectrum of uniaxial crystals $R_{0}$ (Criddle, 1998) and
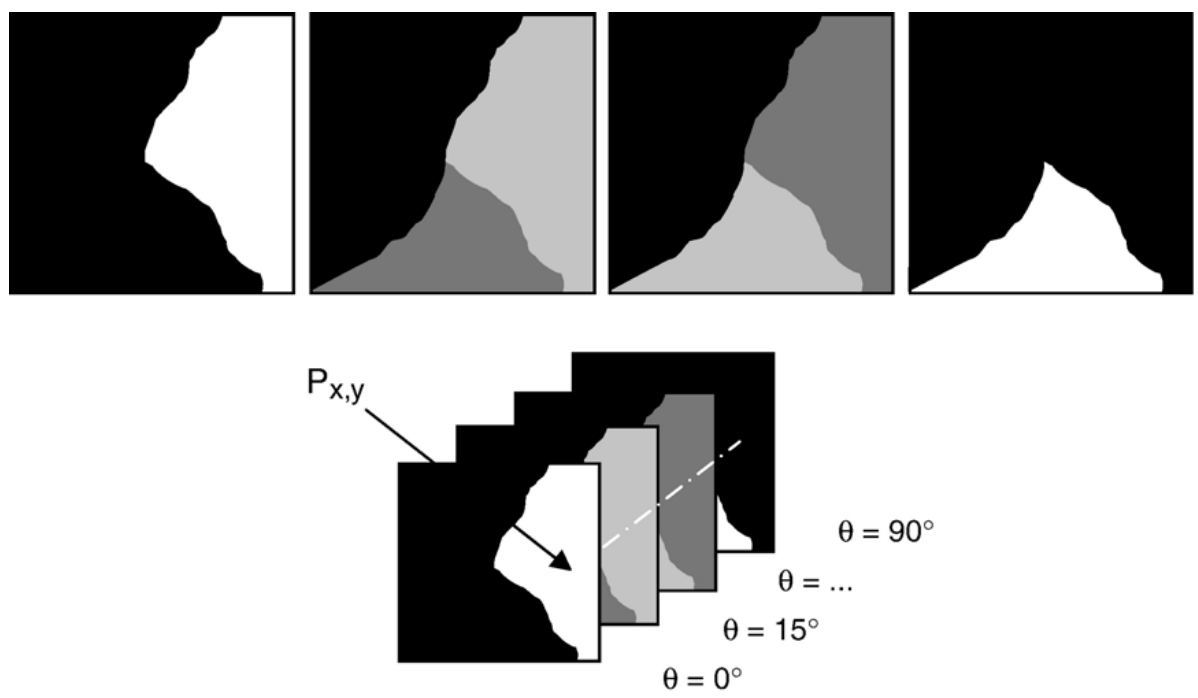

Fig. 5. Schematic representation of imaging under different polarizations $(\theta)$ of the incident beam. Each pixel is characterized by a set of intensities allowing to identify isotropic and anisotropic sections in the sample. 
a

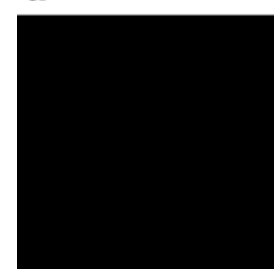

b

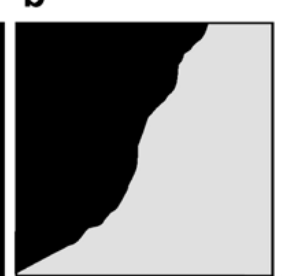

c

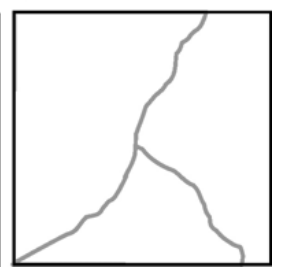

Fig. 6. Three images are computed to synthesize multiradial imaging intensities (at each wavelength): a) the minimum reflectance image; b) the bireflectance image; c) the inverted gradient image. The first ones help to identify mineral species, whereas the last one is used for grain boundary identification.

an indicator of the minimum reflectance $R_{1}$ for biaxial crystals.

A qualitative bireflectance image is computed from the difference between the observed maximum and minimum reflectances:

$B_{(x, y)}^{\lambda}=\left(\max _{\theta}\left\{R_{(x, y)}^{\lambda, \theta}\right\}-\min _{\theta}\left\{R_{(x, y)}^{\lambda, \theta}\right\}\right)$

This is not a quantitative measure of bireflectance because of the unconventional imaging mode and because most often sections do not contain the maximum reflectance spectrum (i.e. section strictly perpendicular to the basal plane in uniaxial crystals), but it reveals which crystals tend to show bireflectance and which appear isotropic (Fig. 6b).

Finally grain boundaries are enhanced in a maximum of gradient image performing the following steps:

- median filtering of every image in order to reduce salt and pepper noise

- computation of a maximum amplitude gradient in every orientation of the polar $(\theta)$ by convolving images with $\begin{array}{rcccccc} & 0 & -1 & 0 & 0 & 0 & 0 \\ \text { the following kernels: } & 0 & 0 & 0 \\ & 0 & +1 & 0 & 0 & 0 & 0\end{array}$

and adding the vertical and horizontal contributions

- computation of a single gradient image containing the maximum amplitude over all orientations of the polar (Fig. 6c):

$$
\nabla_{(x, y)}^{\lambda}=\max _{\theta}\left\{\nabla_{(x, y)}^{\lambda, \theta}\right\}
$$

\subsection{Grey level intercepts for texture analysis}

A very wide set of techniques does exist to address quantitative description of textural features (Tuceryan and Jain, 1998). A first group of techniques relies on feature extraction and so-called blob analysis, whereas another group readily computes spatial statistics (e.g. auto-correlation matrices, sum and difference histograms,...) from grey level or even color images. For the sake of quality control of mineral processing operations and in order to facilitate interpretation in terms of particle populations, blob analysis is usually performed (Lastra et al., 1998).

Blob analysis requires individual phases or individual grains to be perfectly outlined by the image segmentation procedure. Though reasonably easy when dealing with a simplified dual mineralogy (e.g. sulfide/gangue; gold/ gangue), the exact segmentation of an image can be very hard to achieve in ores with complex mineralogy and very often leads to a high rate of misclassification. In case of textures composed of grain intergrowth with identical compositions the challenge of complete grain outlining is almost impossible to achieve. Fig. 7 shows a typical steel texture wherein grain boundaries are very well evidenced by preferential etching at the grain boundary interface. However, thresholding, skeletonization and binary watershed segmentation will be poorly efficient to complete the missing contour information (Beucher and Meyer, 1992; Pirard and Delanaye, 1994). Watersheds, in particular, bring in severe oversegmentation that will have dramatic consequences on computations of average grain size if no posterior processing is applied.

An alternative solution is to drop strictly individual analysis and go for a statistical analysis of grain populations based on linear intercept distributions. This method known as Mean Intercept Length (MIL) has been popularized in earth sciences by Launeau and Robin (1996) to deliver average grain size, aspect ratio and azimuth information from binary images in thin sections. It has been recently extended to grey level intercepts $\left(\mathrm{MIL}^{\mathrm{g}}\right)$ in order

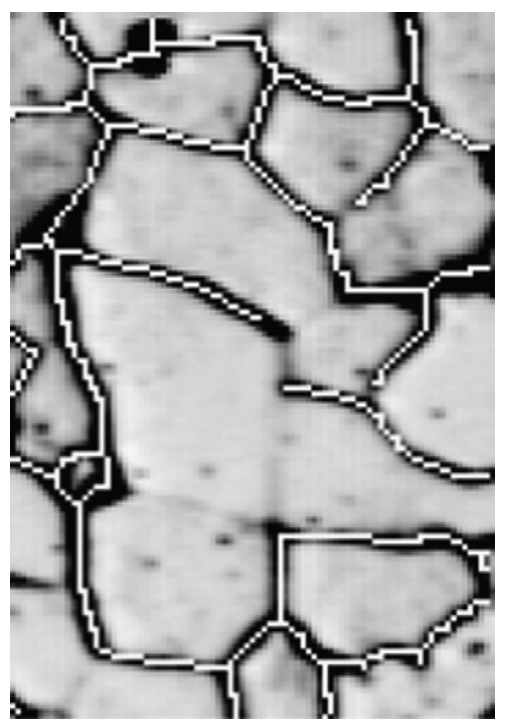

Fig. 7. Etched steel section with superimposed skeletonized boundaries that indicate imperfect segmentation of the grains. 
to reduce artifacts due to small angular rotations of binary images (Lebichot et al., 2005) and take full advantage of grey level information. The technique is applied within a circular mask applied to the image and is being repeated for a discrete number of scanning directions after image rotation is performed using linear grey level interpolation. The major advantages of the grey-level intercept method $\mathrm{MIL}^{\mathrm{g}}$ can be summarized as follows:

- Loss of information due to hard binarization is avoided. The segmentation operation is performed along the intercept scan line and can be based on absolute thresholds, local thresholds or gradient magnitudes.

- Rotation to achieve intercept analysis in any direction takes advantage of the original grey-level information instead of relying on a nearest neighbour analysis in a binary image.

- Grey-level transitions are located in any direction using sub-pixel accuracy (i.e. modelling of the greylevel transition).

Fig. 8 illustrates the principle of grey level intercepts on a multiradial gradient image. In such a case the transitions that are to be searched for are dark-to-bright-todark. This is achieved by thresholding the gradient signal along each scan line and quantifying the amplitude and area of the resulting peaks. Sub-pixel accuracy can be obtained, if required, by modelling the grey level transition with a Gaussian function. In order to make grey level intercept detection even more robust, it is also possible to locate a transition by taking into account the transitions along a series of neighbouring parallel intercepts instead of a single one.
Each analysis along a scanning direction $\alpha$ results in a grey level intercept distribution with a mean value that is directly related to the average grain size in the given direction (Launeau and Robin, 1996). A convenient representation of the results is a rose of intercepts based on percentiles of directional distributions (Lebichot et al., 2005). Fig. 9 represents schematic textures and corresponding roses of intercepts. It should be clear that the rose of intercepts is a global texture characterization tool and as such it is blind to the difference between isometric objects and random (uniform) orientations of anisometric objects (Fig. 9b and d). Mixing of populations induce noticeable differences in quantile contour spacings (Fig. 9a and b).

\section{Texture analysis of iron ore particles}

It has been suggested by several authors that textural features are of critical importance in understanding the behavior of iron ore particles in the sintering process (Vieira et al., 2003). Though there are still many aspects to be debated in order to pinpoint the most critical textural features, it is commonly agreed that the amount of goethite, the porosity of the mineral assemblage as well as the specular or granular character of hematite grains are important characteristics. Iron mineralogy in banded iron formations is limited to three major oxide phases and gangue minerals that are mostly siliceous to argillaceous. Ideal reflectance spectra that do not take into account polishing artifacts, grain size effects, variable compositions and internal reflections show that mineral discrimination is almost immediate from simple thresholding in the central portion of the visible spectrum $(550 \mathrm{~nm}-$

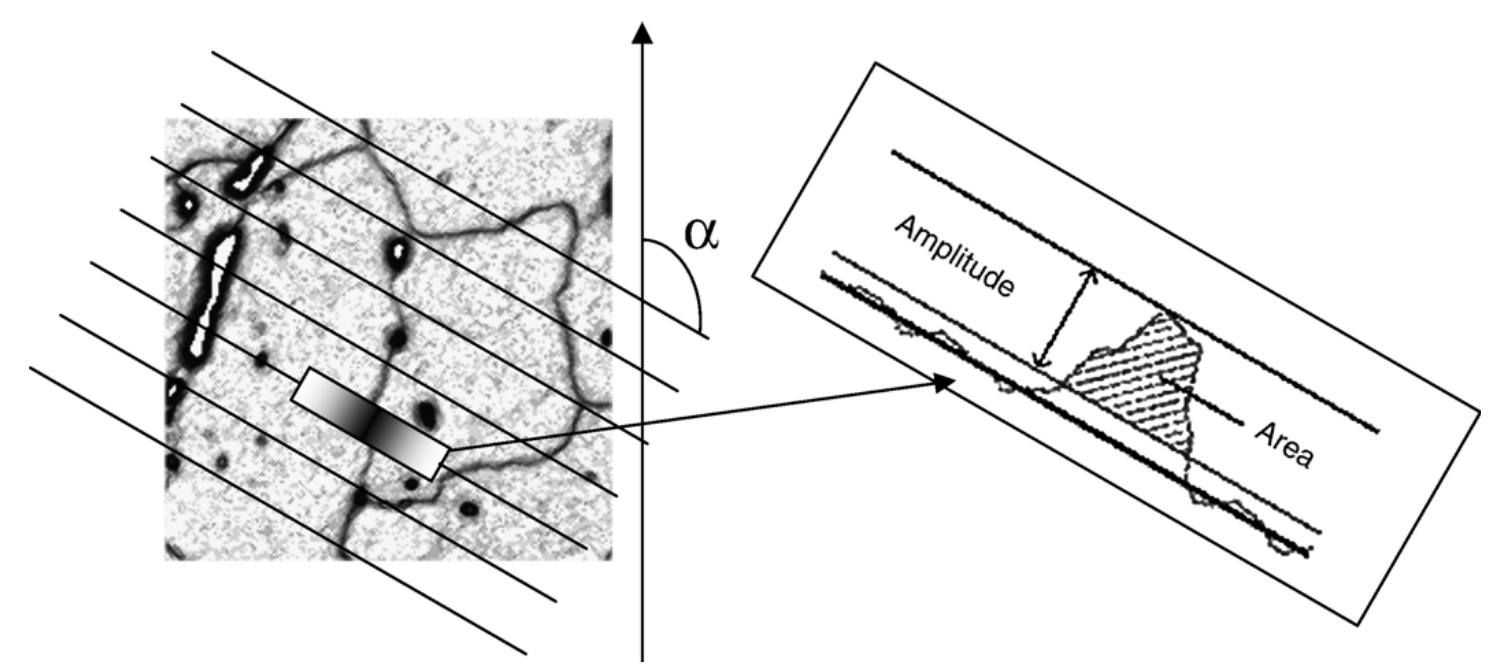

Fig. 8. Illustration of grey level intercept detection along a scan line in direction $\alpha$. A significant gradient is detected whenever the transition peak above a given threshold fulfills both amplitude and area criteria. The background image is a real multiradial gradient image obtained on hematite grains that has been inverted for clarity. 

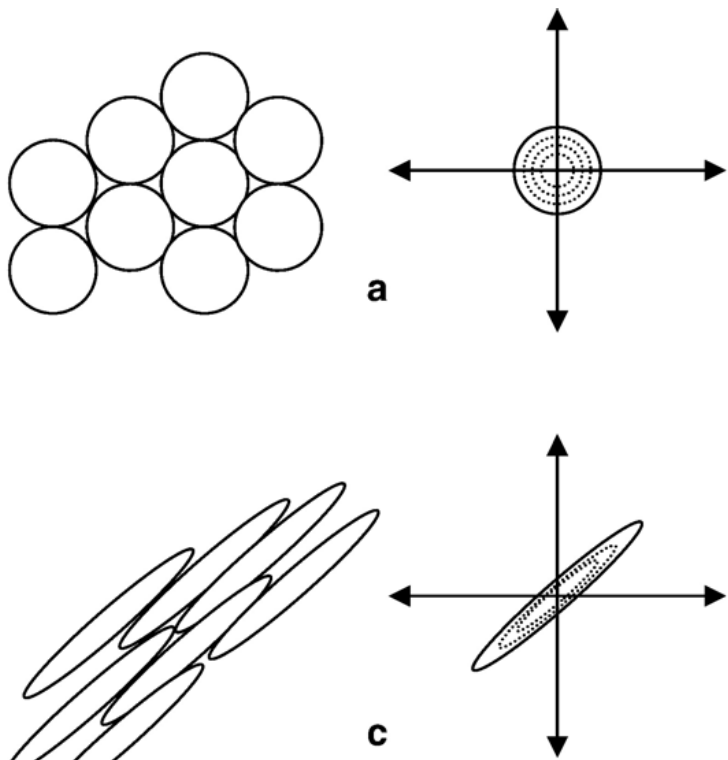

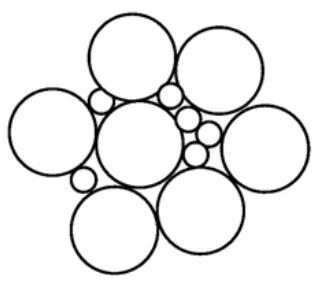

b
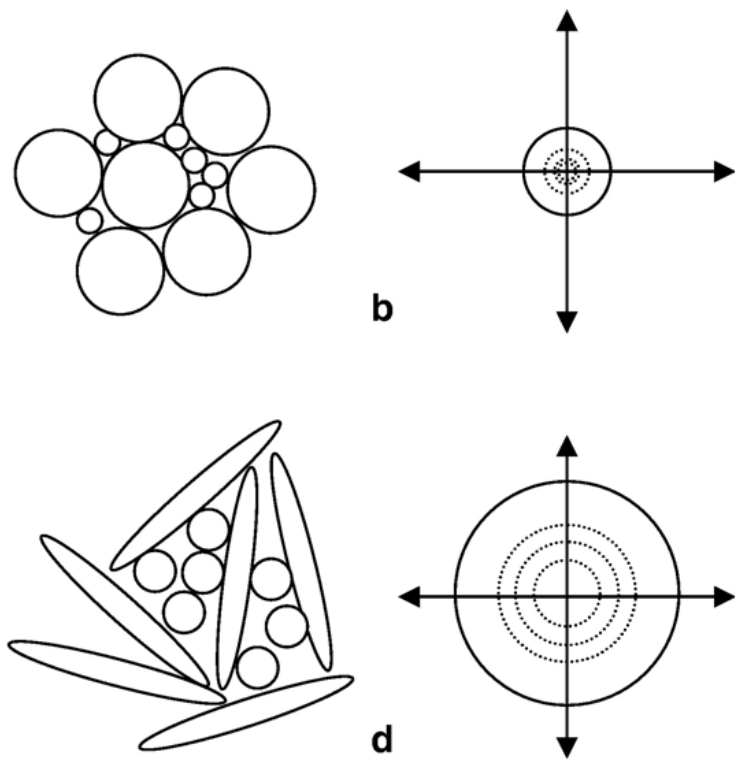

Fig. 9. Theoretical textures and corresponding roses of intercept length with approximate quantile distributions (plain line is the maximum intercept length in each direction).

$600 \mathrm{~nm}$ ) (Fig. 10). In practice, complex limonitic crusts and fine-grained material introduce strong departures from ideal reflectances in hydroxide parageneses, but magnetite as well as hematite have rather stable optical properties that do favor their identification. Fig. 10 shows that hematite has appreciable bireflectance in the order of $4 \%$. Such a difference is perceptible to a trained human eye and proves to be sufficient to discriminate among pixels when using a high dynamic (12 bit) cooled CCD camera as imaging device (Pirard, 2004).

Particles from banded iron formations mineralization have been pictured at $589 \mathrm{~nm} \pm 10 \mathrm{~nm}$ under seven orientations of the polarized incident beam. As explained in the previous paragraph resulting sets of reflectance, bireflectance and gradient images have been computed and used for further analysis. Fig. 11 shows the set of

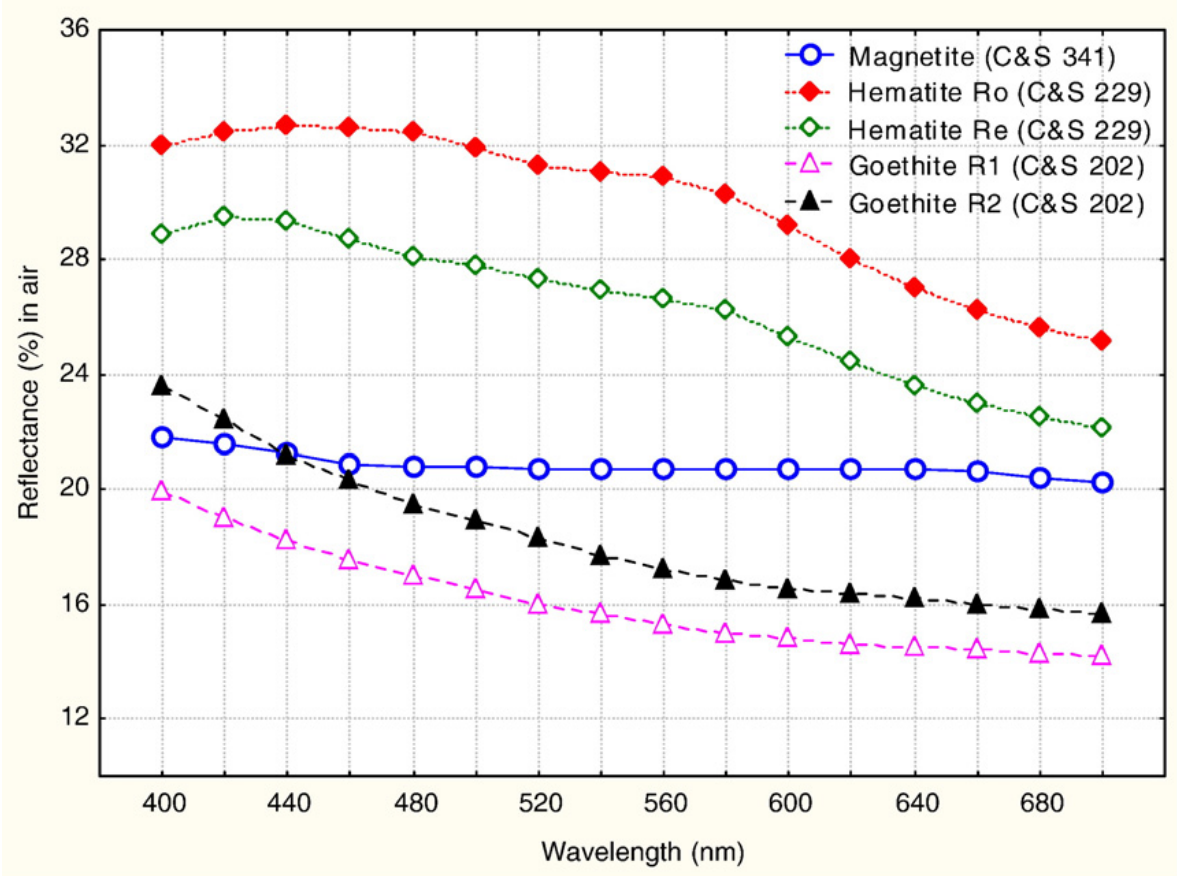

Fig. 10. Reflectance spectra from the QDF database for the main iron oxide species: magnetite (plain line); hematite (dotted line — diamond) and goethite (dashed line - triangle). 
a

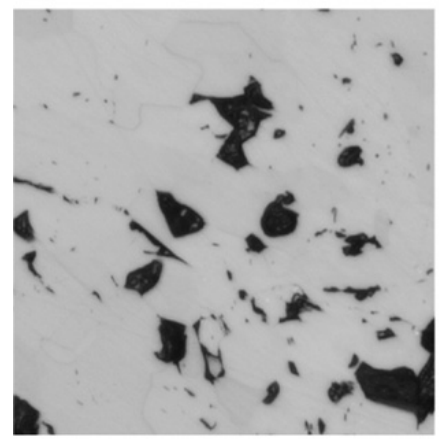

b

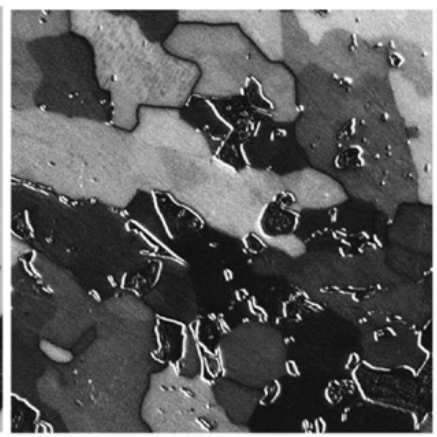

C

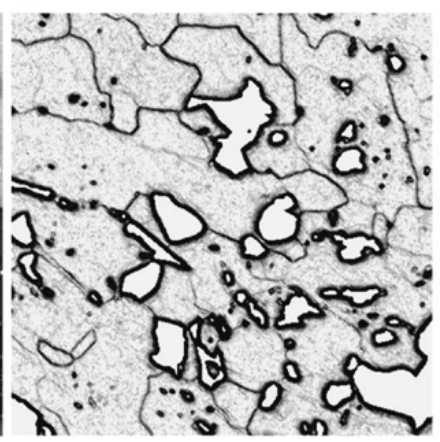

Fig. 11. Minimum reflectance (a) and bireflectance (b) images at $589 \mathrm{~nm} \pm 10 \mathrm{~nm}$ from a granular and porous hematitic particle. Inverted maximum of gradients image of the same scene (c). The grey level histograms of the bireflectance and the gradient images have been stretched for clarity.

images obtained from a typical granular hematitic particle with significant porosity. Thresholding of the reflectance image has been used to separate porosity and magnetite from the dominant hematite.

Finally, prior to the $\mathrm{MIL}^{\mathrm{g}}$ analysis of the gradient image, a porosity mask has been used to remove any grey level transition within the pore structure that could contribute to noise in the rose of intercepts.
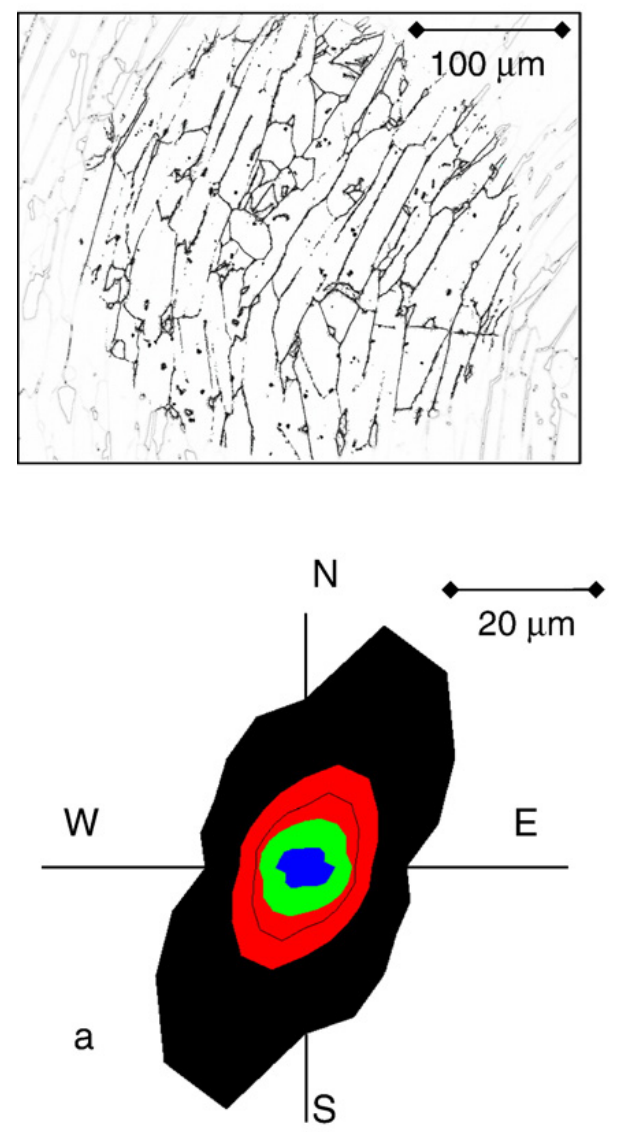

Fig. 12 compares two typical specular and granular textures observed in ore particles and their corresponding roses of intercepts. Both gradient images were analyzed within a circular mask to avoid biased estimations due to the corners of the rectangular frame. The significant transitions detected along the intercept scan lines are marked as dark dots. It appears that grain outlining due to bireflectance gradients is excellent but not at all perfect.
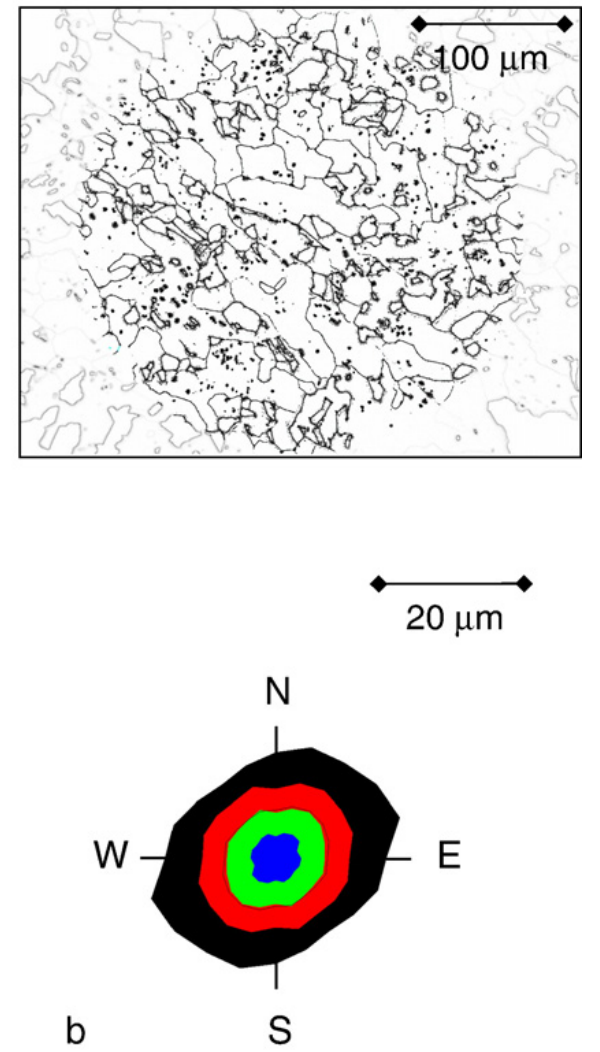

b

S

Fig. 12. Percentile plots $\left(\mathrm{P}_{25} ; \mathrm{P}_{50} ; \mathrm{P}_{75} ; \mathrm{P}_{95}\right)$ of the rose of grey level intercept length obtained for a specular texture and a granular texture. The mean intercept length in each direction is given by the black line. 


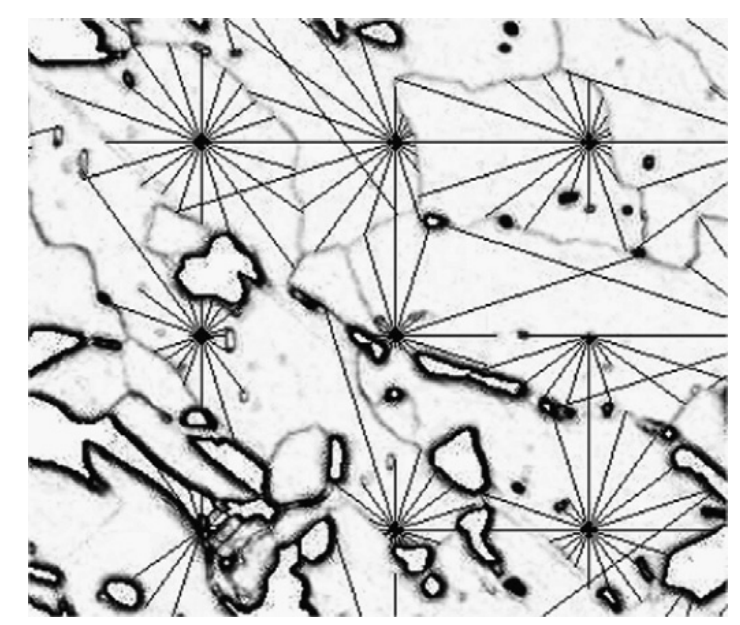

Fig. 13. Star function distribution around selected sampling points in the multiradial gradient image.

Nevertheless, the roses of intercept percentiles for both textures display significant differences. A basic parameter that can be used to classify textured particles based on their rose of intercepts is a simple aspect ratio of the maximum over the minimum mean intercept length:

$\chi=\frac{\max \left\{m\left(L_{\theta}\right)\right\}}{\min \left\{m\left(L_{\theta}\right)\right\}}$

The aspect ratio of the specular texture is 1.56 whereas it is only 1.23 for the granular texture.

If instead of using the ratio of mean intercept length one uses a ratio of higher order percentiles such as $\mathrm{P}_{75}$ the contrast becomes even more striking, ranging from 1.65 to 1.17 .

Aspect ratio together with the absolute value of mean intercept length which gives access to the grain size may serve as a basis for further classification of particles into granulo-textural classes as desired by most iron ore petrographers.

\section{Conclusions and perspectives}

The combination of multiradial imaging and grey level intercept analysis on bireflectant minerals provides quantitative means to classify particles into textural classes. Though the imaging principle could be improved towards true measurement of bireflectance magnitudes that would benefit mineral identification, the grain contrast induced by multiradial imaging is sufficient to bring significant information about grain intergrowth. The intercept analysis proves to be particularly robust against the imperfection of the grain boundary detection procedure, which makes it a very attractive tool as compared to classical blob analysis procedures which are impracticable in many complex ores.

The MIL ${ }^{\mathrm{g}}$ used in the analysis of gradient images has two drawbacks that could largely be improved in future applications. The first one is that it is multiphasic. In other words, the roses of intercepts pool together intercepts running through different mineral species (hematite, magnetite,...) or through the porous structure. Because hematite strongly dominates and pores most often tend to follow the same geometry, the multiphasic roses are still discriminant among textures in the case that has been illustrated in this paper. But, clearly, goethite and magnetite when more abundant will blur the picture and the intercept statistics. A combined analysis of the reflectance image and the gradient image will be required to compute separate roses of intercepts for each phase.

Another improvement in the $\mathrm{MIL}^{\mathrm{g}}$ technique is to go for local analysis. $\mathrm{MIL}^{\mathrm{g}}$ as defined here is a global analytical tool in the sense that a single rose and its aspect ratio are associated to the field of analysis. Should a single particle display mixtures of textures (Fig. 1c, d) or simply bimodal crystal size distributions, than the $\mathrm{MIL}^{\mathrm{g}}$ rose will be blind to it. The computation of local intercepts at random (or systematic) points chosen in the particle is another important improvement leading to the computation of local star area distributions (a local analysis version of the Star Volume Distribution (SVD) introduced by Cruz-Orive et al., 1992). Fig. 13 displays the detection of some local stars in a hematitic texture. The size of polygons delimited by local intercepts is a possible means of texture analysis, but it proves to be too sensitive to poor boundary detection, hence robust measures of star distributions are being developed for efficient use as texture classifiers in future work.

\section{Acknowledgements}

The authors wish to thank Patrick Launeau for the fruitful discussions and the late Alan Criddle for his encouragement to foster the development of quantitative optical ore microscopy.

\section{References}

Adams, B.L., Wright, S.I., Kunze, K., 1993. Orientation imaging: the emergence of a new microscopy. Metall. Trans., A, Phys. Metall. Mater. Sci. 24 (4), 819-831.

Berrezueta, E., 2004. Caracterización de menas mediante análisis digital de imagen. Ph D Thesis, EPM Madrid.

Beucher, S., Meyer, F., 1992. The morphological approach to segmentation: the watershed transformation. In: Dougherty, E. (Ed.), Mathematical Morphology in Image Processing. Marcel Dekker, New York, pp. 433-482. 
Cnudde, V., Masschaele, B., Dierick, M., Vlassenbroeck, J., Van Hoorebeke, L., Jacobs, P., 2006. Recent progress in X-ray CT as a geosciences tool. Appl. Geochem. 21 (5), 826-832.

Criddle, A.J., 1998. Ore microscopy and photometry (1890-1998). In: Cabri, L.J., Vaughan, D.J. (Eds.), Modern Approaches to Ore and Environmental Mineralogy. Short Course Series, vol. 27. Mineralogical Association of Canada, Ottawa, pp. 1-74.

Criddle, A.J., Stanley, C.J., 1993. Quantitative Data File for Ore Minerals, third ed. Chapman \& Hall, London. 635 pp.

Cruz-Orive, L.M., Karlsson, L.M., Larsen, S.E., Wainschtein, F., 1992. Characterizing anisotropy: a new concept. Micron Microsc. Acta $23,75-76$.

Dingley, D., 2004. Progressive steps in the development of electron backscatter diffraction and orientation imaging microscopy. J. Microsc. 213 (3), 214-224.

Fueten, F., 1997. A computer-controlled rotating polarizer stage for the petrographic microscope. Comput. Geosci. 23 (2), 203-208.

Gottlieb, P., Wilkie, G., Sutherland, D., Ho-Tun, E., 2000. Using quantitative electron microscopy for process mineralogy applications. J. Mineral. 52 (4), 24-27.

$\mathrm{Gu}, \mathrm{Y}$., 2003. Automated scanning electron microscope based mineral liberation analysis. J. Miner. Mater. Charact. Eng. 2 (1), 33-41.

Ketcham, R.A., Iturrino, G.J., 2005. Nondestructive high-resolution visualization and measurement of anisotropic effective porosity in complex lithologies using high-resolution X-ray computed tomography. J. Hydrol. 302, 92-106.

King, R.P., Schneider, C.L., 1998. Stereological correction of linear grade distributions for mineral liberation. Powder Technol. 98 (1), 21-37.

Lastra, R., Petruk, W., Wilson, J., 1998. Image analysis techniques and applications to mineral processing. In: Cabri, L.J., Vaughan, D.J. (Eds.), Modern Approaches to Ore and Environmental Mineralogy. Short Course Series, vol. 27. Mineralogical Association of Canada, Ottawa, pp. 327-366.

Launeau, P., Robin, P.-Y.F., 1996. Fabric analysis using the intercept method. Tectonophysics 267, 91-119.
Lebichot, S., Dislaire, G., Pirard, E., Launeau, P., 2005. Grey level intercepts distributions and grain size estimation. Proc. 8th Int. Congr. on Stereol. and Image Anal., Zakopane, PL, pp. 102-110.

Lin, C.L., Miller, J.D., 1996. Cone beam X-ray microtomography for three-dimensional liberation analysis in the 21 st century. Int. J. Miner. Process. 47 (1-2), 61-73.

Mermillod-Blondin, R., 2005. Influence des propriétés superficielles de la pyrite et des minéraux sulfurés associés sur la rétention de molécules organiques soufrées et aminées: application à la désulfuration environnementale. Ph D Thesis, INPL Nancy.

Petruk, W., 1988. Automatic image analysis for mineral beneficiation. J. Met. 40, 29-31.

Pirard, E., 2004. Multispectral imaging of ore minerals in optical microscopy. Mineral. Mag. 68, 323-333.

Pirard, E., Delanaye, A., 1994. Conditional watershed segmentation for grain boundary reconstruction. Acta Stereol. 14, 23-28.

Prior, D.J., Boyle, A.P., Brenker, F., Cheadle, M.C., Day, A., Lopez, G., Peruzzi, L., Potts, G., Reddy, S., Spiess, R., Timms, N.E., Trimby, P., Wheeler, J., Zetterstrom, L., 1999. The application of electron backscatter diffraction and orientation contrast imaging in the SEM to textural problems in rocks. Am. Mineral. 84, 1741-1759.

Russ, J.C., Dehoff, R.T., 2001. Practical Stereology, second ed. Plenum Press. 307 pp.

Tuceryan, M., Jain, A.K., 1998. Texture analysis, In: Chen, C.H., Pau, L.F., Wang, P.S.P. (Eds.), Handbook of Pattern Recognition and Computer Vision, second ed. World Scientific Publishing Co. Pte. Ltd., pp. 207-248.

Van der Pluijm, B.A., Marshak, S., 1997. Earth Structure - An Introduction to Structural Geology and Tectonics. WCB-McGrawHill. 495 pp.

Vieira, C., Rosiere, A., Pena, E., Seshadri, V., Assis, P., 2003. Avaliaçao tecnica de minerios de ferro para sinterizaçao nas siderurgicas e mineraçoes brasileiras: uma analise critica. Rev. Esc. Minas Ouro Preto 56 (2), 97-102. 